\title{
Wlan Based Positioning With A Single Access PoINT
}

\author{
Vitaly Romanov and Giancarlo Succi \\ Innopolis University, Innopolis, Republic of Tatarstan, Russia
}

\begin{abstract}
WLAN has lately been applied to the problem of mobility tracking and behavior analysis. To further the development of the studies in this direction the positioning system that can perform on the network side with minimal human participation is needed. One of the current limitations is the requirement on the number of reference signal available. Thus, methods that require fewer reference signals for positioning are needed. This paper provides the comparison of WLAN based positioning methods that can operate with a single $A P$.
\end{abstract}

\section{KEYWORDS:}

Localization, Wireless, WiFi, Mobility Tracking, Mobile Users.

\section{INTRODUCTION}

WiFi has been used as an additional infrastructure for positioning systems for a long time. In an indoor environment, it is crucial for precise localization. Recently, we have seen the interest in using WiFi as a tool for analyzing mobility of people $[1,4,18,19]$. These works try to track the movements of a person and potentially deduct additional information either about the place or the person based on the movement history of an individual or a group. Indeed, the mobility tracking exists beyond $\mathrm{WiFi}$ for a long time [27]. Well developed techniques to process the movement of the people and detect the common mobility pattern exist [5]. Although we see the beginning of the use of WiFi as a mobility tracking tool, there are some limitations. Some techniques are solely based on tracking the occupancy of wireless access points (APs), and some look into proximity based on two devices sensing each other. Both of these approaches are limited and it is hard to relate granular information about location semantics and the actual position of a person.

Mobility tracking relies on the ability of a sensor network to infer the location of people, and conventional positioning systems are not capable of doing that. It is possible to use cellular networks for this task, but due to the large coverage area precision of cellular based position suffers [22]. The latest development in crowd mobility tracking with WiFi relies on special broadcast probe packets transmitted by mobile devices. Most of modern APs can intercept these packets and infer the approximate position of a mobile device based on RSSI. The most advanced and readily applicable approaches rely on the plethora of reference signals. Conventionally this means the mobile device can see the signal strengths of many APs, but in our context, this implies that several APs intercept the probe packet. The most significant limitation here is that not all areas are covered with dense wireless networks where one position is several wireless routers already observe space. Moreover, the need for APs continuously monitoring the environment prevents them from serving their primary purpose, and thus one cannot rely on the probe packet 
being intercepted by many APs at once. What is needed in this case is the ability to infer the location of a person based on few location signals available.

Classical approaches for radio-based positioning use such techniques as triangulation or trilateration to identify the location of a device, but they also require at least three reference signals available, and they work under the assumption of free space radio signal propagation. Other widely used methods for positioning with WiFi rely on the received signal strength indicator (RSSI). In this case, the location on the map is associated with the signal strength that is observed in this particular point of the map. There plenty of methods that rely on this approach proposed, they have higher accuracy even in the environments prone to multipath signal propagation, but they also require more computational resources.

Techniques based on RSSI proliferated in WLAN-based positioning since the information about the received signal strength is readily available on any modern wireless access point. These approaches have found the most application in an indoor environment where WiFi networks are ubiquitous. The methods that rely on the signal strength are often referred to as fingerprint techniques since they create an RSSI fingerprint for every stored location on the map.

Indoor finger print-based positioning is usually performed with multiple APs, but this way is not suitable for mobility tracking, as we discussed before. Very limited number of works have considered the positioning quality of their systems with only one or two APs in operation, especially in the indoor environment where triangulation and trilateration techniques are hardly applicable. Our work is dedicated to reviewing the methods that can work with few APs available. Our contribution is the following:

1. systematizing the knowledge about the techniques that use only few APs for positioning;

2. providing the comparison of these techniques with respect to parameters listed in section 4 .

The rest of the paper is organized as follows. In section 2 we overview related work. In section 3 we formulate the problem of positioning in WiFi networks and discuss signals used for positioning and limitations that emerge when positioning with a single AP. In section 5 we classify existing techniques for positioning with one AP. Section 6 concludes the paper.

\section{RELATED WORK}

Many attempts to systematize the knowledge on WLAN-based positioning were done in the last years $[2,8,9,15,17,22-24]$. Different parameters of positioning systems were considered for these careful studies, from the precision of localization to sensor fusion and energy efficiency. However, these studies did not consider the behavior of positioning systems in the scenario of a limited number of reference signals available. Now we are going to overview existing surveys on WLAN-based positioning.

Positioning in WLAN is related to other forms of wireless positioning, more precisely, positioning with radio signals. Liu et al. (2007) provided a general review of methods for localization in wireless networks and performed their comparison concerning accuracy, precision, complexity, scalability, robustness, and cost [13]. 
Farid et al. (2013) considered only indoor positioning techniques [6]. They included recent work and performed the comparison of positioning methods using other criteria, such as coverage provided by the system, power consumption, and susceptibility to multipath.

Quoc Duy Vo and Pradipta De (2016) compared existing wireless positioning techniques for outdoors concerning accuracy and power consumption [22].

Until recently, there were two types of information that a client or an AP could measure during communicating in WLAN. The first is the RSSI and the second is temporal delay. With the introduction of MIMO (Multiple Input Multiple Output) in IEEE 802.11 standard, it became much simpler to obtain the information about the direction of the incoming signal.

The initial attempt to create indoor positioning system with WiFi could be classified as timebased or signal strength-based. Although time-based methods often do not allow using -the-shelf hardware, Makki et al. (2015) explored the opportunity of positioning using time measurements in $\mathrm{WiFi}$ and discussed existing ways to overcome the issues with resolution and the prospective opportunities of time-based positioning in WLAN [15].

Suining He and Gary Chan (2016) considered only fingerprinting methods for localization [8]. They noted the use of spatiotemporal patterns, collaborative localization, and motion assistance in recent papers. Additionally, they reviewed different techniques for reducing the site survey, calibrating the system for heterogeneous devices, and performed the comparison of many methods using various criteria.

Although RSSI-based methods deal with the problem of multipath, they require the knowledge of space configuration and the presence of signal strength map, which takes a lot of time and resources to collect. Mahtab Hossain and Wee-Seng Soh (2015) decided to look into calibrationfree indoor positioning methods, which do not require extensive map collection or even the precise knowledge of the floor plan [9]. For comparing these calibration free methods, they considered map requirements, the necessity of the initial location fix, the amount of user participation, and the need for additional sensors, such as inertial sensors.

Radio map estimation is a challenging problem and vital for fingerprint based positioning. Yiu et al. reviewed different approaches to radio map construction, and the radio map is one of the most important aspects of positioning with limited information [2]. The more accurate the radio map is the better are location estimates. They studied the effect of the AP density and the effect of outdated radio maps.

Modern trends in positioning systems include sensor fusion and device free positioning. Xiao et al. performed the comparison of methods for device-free and crowd-sourced assisted localization [23]. They performed their comparison in terms of accuracy, cost, energy efficiency and technical difficulty.

The difference of our review is that we consider only positioning techniques that require only a single active AP to localize the client. Intuitively, if a method achieves reasonable localization error with a single AP, it's results are likely to improve when scaled to several APs. Papers covered in this review were not considered in previous surveys. Our contribution is in aggregating the information about this subtype of positioning methods and providing their comparison. 


\section{Problem Formulation}

\subsection{Positioning procedure}

The goal of positioning is to identify the location of a user relative to a set of base stations (BS). In the case of WLAN-based positioning, the user is a network client, and APs serve as BS. Conventionally, two types of positioning approaches are distinguished: deterministic and probabilistic [8]. Deterministic algorithms use some similarity or distance metric to compare observed signals with a prerecorded database. In this case, the positioning procedure becomes an optimization problem

$$
\hat{l}=\operatorname{argmin}_{l} \operatorname{SIM}\left(\mathbf{s}, \mathbf{s}_{l}\right)^{b}
$$

where SIM is a similarity function, and $\mathrm{b}=1$ when $\operatorname{SIM}\left(\mathrm{s}, \mathrm{s}^{\prime}\right)$ increases as $\mathrm{s}^{\prime} \rightarrow \mathrm{s}$ and $\mathrm{b}=-1$ if otherwise. Here, 1 denotes the location, and $\mathrm{sl}$ - the signal associated with this location.

On the other hand, the probabilistic approach employs maximum likelihood (ML) technique where a probability distribution ties together the signal space and locations on the map and the goal is to maximize the conditional probability

$$
\hat{l}=\operatorname{argmax}_{l} \mathbf{P}(l \mid \mathbf{s})
$$

These two approaches constitute the basis for building positioning algorithms and the papers considered further use either of them.

Besides choosing the location estimation method, it is essential to select an appropriate set of signals. In the next section, we will discuss the types of measurements that are used for positioning in WLAN.

\subsection{Signals for positioning in WLAN}

Measures that are used for positioning in WLAN include RSSI, time or phase, the angle of arrival (AoA), and channel state information (CSI). In this paper, we refer to those as location signals, and their properties are described below.

RSSI measurements are prone to high variance especially in indoor environments [3]. Although RSSI was initially adopted for positioning due to its indifference to multipath, latest work shows that it suffers from non-stationary variance [10]. Nevertheless, positioning with RSSI in WLAN is the most common technique to

date.

Positioning in WLAN can be done using time or phase measurements. The simple idea is that these measurements can be converted into the distance that is further used to localize a user. Although less sensitive to noise, time intervals are harder to measure using o_-the-shelf WLAN adapters due to internal clock resolution and the radio bandwidth of the received signal [15]. It was estimated that with the standard bandwidth of $40 \mathrm{MHz}$ the inaccuracy of time measurements results in localization errors around 7 meters [20]. This number can be improved by applying special techniques. 
CSI describes the state of the communication channel and provides rough infor- mation about channel impulse response. CSI is represented by phase and amplitude of the received signal in the frequency domain. The IFFT of CSI gives the notion of delays of different multipath components. Recent work treats it as a promising localization signal [3]. Currently, CSI is not available by default, and the driver for a wireless adapter should be modified to obtain the access to these measurements.

Methods for positioning described in this review use at least one of the signals above to infer user's location and some of them use their combination to improve the precision.

\subsection{Positioning with a single active AP}

In general, the level of confidence for estimated location is low when positioning with a limited number of APs. For an indoor environment, this problem can be addressed by considering the joint distribution of RSSI and the location on the map. The presence of obstacles on the way of WiFi signal propagation, like walls, create an irregular RSSI pattern across the area. Some patterns make it easier to infer the true location. The distribution of average RSSI across space is called coverage map. Techniques that utilize such coverage maps are usually called scene analysis techniques [13]. It is worth noting that the irregularity of a coverage map highly depends on the configuration of the environment, and in open spaces such maps do not provide significant benefits over other less memory intensive techniques such as statistical propagation models.

Another approach is related to the use of triangulation or triangulation. The position of a client can be identified when sets of distances and/or AoAs are known. With the introduction of MIMO in the current IEEE 802.11 standard, each com-patible AP is equipped with multiple receiver inputs. This receiver antenna array can be used to infer the relative position of a user and the AP. For simplicity, we further refer to triangulation and triangulation techniques under a common term of triangulation.

We were able to discern three classes of positioning techniques with a single AP: (i) triangulation (ii) triangulation and dead-reckoning (DR) fusion (iii) scene analysis and dead-reckoning fusion.

The first class refers to techniques that rely on the one-time measurements, i.e. the location can be inferred from a single sample of all necessary parameters. The second one includes methods with similar positioning principles but relies on dead-reckoning for obtaining the final location estimate. The third - uses RSSI and coverage map together with dead-reckoning for localization.

\section{COMPARISON CRITERIA}

We choose to compare methods presented here using the following attributes. First, we distinguish the use of different positioning approaches, which define the set of used localization signals and processing techniques.

Often, the positioning system becomes harder to implement when there is a need for additional sensors. Therefore, we try to identify the extent to which a particular positioning procedure uses other measurements. 
Some approaches must take advantage of low-level information from the physical layer of the communication protocol (PHY). This is often associated with particular modification and requires additional work during deployment.

The sampling rate (SR) is often crucial for positioning, especially when dead- reckoning is used. High SR is associated with higher energy consumption and com-munication overhead.

The amount of user involvement in the positioning process differs for presented positioning methods. The necessity of additional computation on the side of the user requires both energy resources and specialized software, hindering seamless deployment of the positioning system.

One of the most critical factors for deployment is the necessity of extensive site survey. While it is nil for triangulation techniques, the presence of fingerprint database is the requirement for scene analysis methods.

Further, we are going to compare different techniques concerning these parame-ters. All information is taken from the reports produces by authors of corresponding methods, and we specify when we make our own notes and conclusions.

\section{Positioning TechniQues}

\subsection{Triangulation}

The class of triangulation techniques includes methods that rely only on explicitly measured parameters and exclude the need for tracking. Thus, these techniques allow for positioning at any moment of time without the knowledge of previous locations. Also, they use only measurements for distance and angles.

CHRONOS The positioning system, named Chronos, designed by Vashisht etal. (2016), is based on commodity wireless adapters and allows localization with a decimeter level of precision [21]. It is claimed to be the first system that per-forms positioning with such accuracy without external sensors. The core idea of the method is to estimate distances between antennas of the AP and a client using absolute ToF.

Chronos uses commodity hardware Intel 5300 with a modified driver. The use of ToF is associated with a series of issues that were discussed in the section 3.2 but authors were able to find a way mitigate these limiting constraints.

Authors substantiate the claim that the phase of the zeroth sub-carrier in OFDM spectrum of WLAN transmission is equal to the true value of the phase, which allows eliminating additional phase shift due to packet detection delay. Thus, ToF can be computed from the expression [21]:

$$
\phi_{i, k}=-2 \pi f_{i, k} \tau \bmod 2 \pi
$$

where $\tau$ is the ToF, and $f_{i, k}$ is the frequency of the sub-carrier $k$ in the frequency band $i$, and $\varphi_{i, k}$ is measured by the receiver. Obviously, such an equation has an infinite number of solutions for $\tau$. The unique solution can be found from a system of equations 
that uses frequencies for different channels. For this reason, the localization procedure involves channel sweep.

The problem of multipath is dealt with using channel state information (CSI), which allows separating the component of the direct path from the rest. The final location of a user is found by solving a quadratic optimization problem. The goal of such optimization is to minimize the difference of distances between a user's assumed location and the AP, and distance estimations calculated from ToF mea- surements.

In the case of Chronos, the AP should have the modified driver installed. This driver provides access to physical layer information and enables the AP to drive frequency hopping procedure. The distance is estimated from the system of signal phase equations and does not require an onboard high-precision clock. In the case of LOS, 95\% of ToF estimation error is contained within $1.96 \mathrm{~ns}$, and the positioning error is under $2 \mathrm{~m}$ in $90 \%$ of cases. One of the essential features of Chronos is its ability to produce confident location estimation after a single scan.

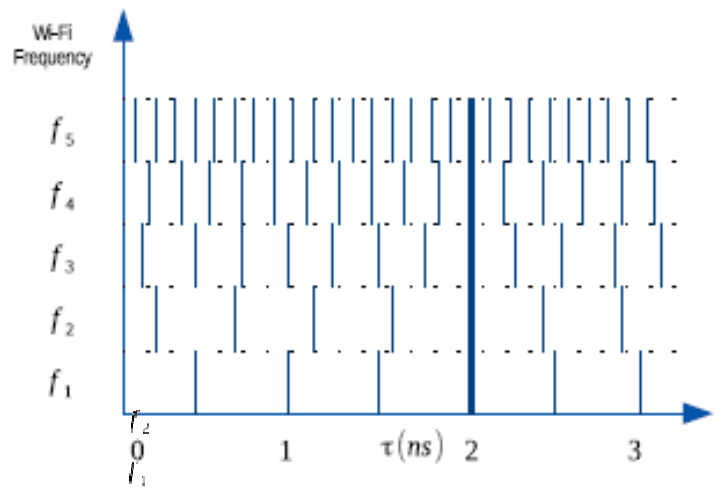

Fig. 1. Example demonstrates the process for solving the ToF based on measured phase. In each channel, there is a set of periodic solutions. The answer corresponds to the time $\tau$ when solutions for all the channels match [21].

The success of Chronos arises the question of the impact of this positioning procedure on the network throughput. It was shown that single localization at- tempt that involves multiple channel sweeps reduces throughput of the network by approximately $5 \mathrm{MBits} / \mathrm{s}$ for the period of localization, which lasts about $84 \mathrm{~ms}$.

Triangulation with passive sensors Another triangulation approach was pro- posed by Kraxberger et al. (2010) [11]. They developed a method that allows identifying the location without active collaboration from a user. In their scheme, there is a single active AP that communicates with the client, and several passive sensors. The job of sensors is to monitor the wireless channel and intercept packets transmitted by the client. The RSSI of intercepted packets carries the information about the distance to the client, which can be extracted using Two-Way-Ground propagation model. After distances between the client and three of the sensors are estimated, triangulation is applied.

This technique does not require any modification of hardware or protocol logic and can be used with any off-the-shelf wireless adapter and AP. However, this method relies only on the statistical propagation model, which can be highly imp-precise in 
certain environments. Thus, the effect of multipath is modeled only ap-proximately. The authors do not evaluate the average accuracy of their method, making it difficult to perform the comparison with different techniques.

\subsection{Probabilistic Models}

Figuera et al. presented their positioning algorithm based on a non-parametric statistical test [7]. Their methods concerns classical fingerprint-based positioning algorithms. They observed that classical approaches mostly make use of the average signal strength at some location on the map. Intuitively, some certainty in the result of a positioning system is needed. Moreover, different positioning systems are oftencompared concerning average positioning error, but the difference in the localization quality is more likely to be a random value and should be subjected to the statistical test. In their work, Figuera et al. introduced a non-parametric statistical test based on bootstrap that provides a certainty measure of a single performance indicator. Also, they have introduced the notion of bias and uncertainty as additional merits of positioning quality.

One of the problems of existing models for positioning with RSSI is that they are imprecise. Very often, the RSSI value is modeled as normally distributed around the mean value. Empirical study showed that this is not necessarily the case [14]. Moreover, while it is hard to capture the distribution of RSSI model correctly, a non-parametric approach does not need this information and can give proper results without knowledge. The use of bootstrap approach allowed to narrow the confidence interval for positioning.

Figuera et al. performed a comparison of different positioning algorithms, and, among else, the comparison of the number of APs for positioning. They observe that the use of at least two APs significantly reduces the positioning error, and this result is statistically significant. The positioning accuracy with just one AP is 5 meters.

Youssef et al. performed an analysis of probabilistic approach for positioning with WLAN [25].

$$
\hat{l o c}=p(\operatorname{loc} \mid R S S I)
$$

They Considered positioning problem as a MAP estimate of the location. To calcu- late the MAP estimate one needs to know the prior on possible locations $p(l)$. They considered different priors on locations and analyzed the impact on positioning precision. The finding was that in some scenarios the knowledge of prior location could reduce the positioning error from $20 \mathrm{~m}$ to $2 \mathrm{~m}$.

\subsection{Distance-based}

Li et al. conducted an unusual study of privacy of WiFi positioning systems [12]. In their work, they designed a system that can adequately protect the privacy of a positioning service user and the intellectual property of the service provider. Alongside, they presented a new positioning approach. The positioning problem is 
distance based. The localization is performed using a sort of nearest neighbor solution. The mobility prediction model performs additional filtering. In this model, the floor plan is represented as a graph, and the one can model the movements in the environment as a random walk on that graph.

The quality of the algorithm was evaluated on several test beds. The overall results show that the average positioning quality with one AP is close to $6 \mathrm{~m}$, and it further decreases as the number of available APs grow.

\subsection{Scene analysis and mobility model fusion}

Zaruba et al. (2007) presented a method for indoor localization with a single access point that uses both scene analysis and dead-reckoning [26]. By combining these two approaches, they were able to achieve average positioning accuracy of $2 \mathrm{~m}$.

Before the positioning procedure can be used, one needs to calculate signal coverage map. To achieve this goal, the accurate floor plan is obtained, and then parametric ray-tracing is performed. By simulating the propagation of radio waves, one can predict the observed RSSI at a given point on the floor plan. To simplify and enhance the ray-tracing process, the passing and reflection coefficients are treated as unknown parameters. Their values obtained by numerically solving the minimization problem where the average squared difference between observed and estimated RSSI measurements serve as the cost function.

Positioning with fingerprints generated in such a way produces a set of possible locations that is too large in general. However, the use of dead-reckoning together with analytical movement model allows filtering the most unlikely positions of users. In this method, no additional sensors on the device are used, and analytical move- ment model is employed. The consecutive samples of this model follow Markov assumption. User's positions are processed with particle and Bayesian filters to increase the positioning accuracy.

This positioning technique handles the problem of the multipath well and re- quires no tampering with the WLAN communication protocol. However, the de-ployment is mostly hindered by the necessity of detailed floor plan. Moreover, the ray tracing procedure is merely an approximation of the real radio wave propagation process and inherently contributes to the final positioning error.

\subsection{Triangulation and dead-reckoning fusion}

In this class, the difference from the triangulation techniques is that some parameters are not estimated after single sampling is performed. Instead, necessary information is accumulated through the extent of time before the estimation can be produced.

CUPID Sen et al. (2013) designed a method that relies on measurements from client's device and a single AP and does not require extensive site surveying [20]. Their method uses distance estimated from a propagation model, and AoA obtained through deadreckoning. The median localization error of the proposed technique is $5 \mathrm{~m}$. They successfully utilize information about multipath components, provided by CSI, and 
knowledge of user mobility to implement a system that works on commodity wireless cards.

In the presence of multipath, the value of RSSI can deviate from the expected value, and statistical propagation models are usually unable to reasonably describe this deviation, especially when the direct path component is not the strongest in the delay profile. On the other hand, when multipath components are filtered, the propagation model can be of greater use. Thus, Sen et al. use the energy of direct path (EDP) in conjunction with propagation model to estimate the distance between AP and the client. EDP is easily obtained once CSI is available.

The AoA is estimated with the facilitation of dead-reckoning. The true AoA can- not always be determined even when an AP with MIMO capabilities is used. In some scenarios, the strongest energy is contributed by a multipath component, drawing conventional methods for determining the direction of arrival of the strongest sig- nal useless. A pseudo-spectrum is usually constructed outlining the dependence of RSSI from the AoA, and the angle that yields the peak energy value is chosen. If local maxima are considered, the procedure will result in the set of possible angles that needs further refinement. The possible solution is to use movement tracking with accelerometer and estimate traveled distance. As a result, a triangle can be constructed with two sides measured with propagation model, and the third - estimated through dead-reckoning, as shown in Fig. 2. Thus, angles from the set of possible solutions can be tried. After identifying the AoA and the distance, user's position is estimated.

Fig. 2. Angle of direct path corresponds to the triangle with sides $d A, d B$ and $p A B$. When signals from multipath components are tried, the angle does not fit this triangle [20].

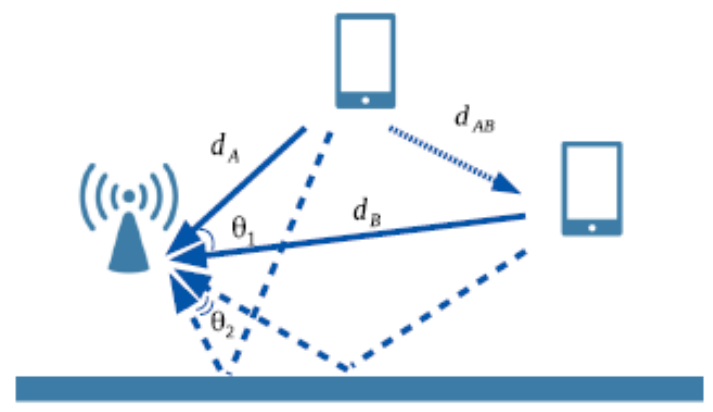

This method introduces a calibration-free approach to local area positioning. It is claimed that the positioning procedure was designed with energy efficiency in mind and probing the client's location with a high rate is not required. On the other hand, the accuracy of DR highly depends on the sampling rate of the device's internal sensors. The method assumes the presence of direct path in the CSI. Authors assert that measurements of EDP lower than $12 \mathrm{~dB}$ are unreliable and should be discarded. This approach can be extended to use several AP with a successful decrease of localization error.

SAIL The improvement of the previous approach was introduced by Mariakakis et al. 
(2014) [16]. The location is determined using distances measured through ToF and DR with the resulting median positioning error of $2.3 \mathrm{~m}$. They take advantage of CSI and user mobility to increase the performance of measurements, and device a heuristic for estimating absolute heading.

The ability of the AP to measure the delay between packet departure and ACK signal arrival lies in the foundation of this technique. The precision of time measure-mends limited by $40 \mathrm{MHz}$ bandwidth is $25 \mathrm{~ns}$. This precision can be enhanced by evaluating the relative time delay of signal arrival to different antennas. Addition- ally, the incorporation of human mobility and channel coherence property allows for the further decrease of positioning error.

Authors improved DR procedure by increasing the accuracy of the mobile de- vice's inertial system. Driven by the goal to estimate the absolute heading, they develop a procedure to switch between readings from magnetometer and gyroscope depending on the current conditions. As a result, the average heading estimation error dropped from 27.4 to 5.9 degrees.

Eventually, the location was determined by constructing a triangle with two sides estimated using ToF, and the third - using DR. The number of possible triangle orientations was reduced by considering the absolute heading.

In this method, the accuracy of CSI measurements is increased by exploiting the channel coherency. Thus, the measurements are performed several times consecutively. This results in a small communication overhead.

\section{CONCLUSION}

We have reviewed methods for positioning in WLAN with a single AP. Conventionally, single AP provides only limited information that is not suitable for confident localization. Moreover, techniques for identifying both the distance and the direction to the user are constrained by the presence of multipath.

The papers described here address the problem of multipath and propose meth- ods that allow precise positioning in areas where it was not feasible before. We compared presented techniques according to the number of criteria that show the trade-off between the complexity of deployment and the complexity of positioning procedure.

Most of the introduced algorithms belong to the class of calibration-free techinquest, which presents a significant advantage for deployment. All methods use commodity hardware, and some of them require the access to CSI provided by the driver.

The method presented by Zaruba et al. (2007) and Kraxberger can work with any hardware without limitations. Chronos, CUPID, and SAIL require WLAN stan- dard $802.11 \mathrm{n}$ and above in conjunction with a modified driver. Although Chronos 
International Journal of Wireless \& Mobile Networks (IJWMN) Vol. 10, No. 3, June 2018

Table 1. Comparison of techniques for positioning with a single AP. FP - fingerprinting, SR - sampling rate, IMU - inertial measurement unit, PHY - physical layer.

\begin{tabular}{|c|c|c|c|c|c|c|c|}
\hline Name & $\begin{array}{l}\text { Pos. } \\
\text { Method }\end{array}$ & $\begin{array}{l}\text { Extra Sen- } \\
\text { sors }\end{array}$ & PHY Info & High SR & $\begin{array}{l}\text { User Par- } \\
\text { ticip. }\end{array}$ & \begin{tabular}{|l|} 
Comm. \\
Overhead
\end{tabular} & \begin{tabular}{|l|} 
Median \\
Error
\end{tabular} \\
\hline Chronos [21] & Trilat. & None & Yes & No & \begin{tabular}{|l|} 
Modified \\
driver \\
required
\end{tabular} & $\begin{array}{l}\text { Location } \\
\text { fix in } 84 \\
\mathrm{~ms}\end{array}$ & $64 \mathrm{~cm}$ \\
\hline Zaruba[26] & $\begin{array}{ll}\text { DR } & \text { as } \\
\text { sisted } & \\
\text { FP } & \end{array}$ & None & No & Yes & $\begin{array}{l}\text { Measures } \\
\text { RSSI }\end{array}$ & None & $2 \mathrm{~m}$ \\
\hline CUPID[20] & \begin{tabular}{|ll} 
DR & as \\
sisted & \\
trilat. & \\
\end{tabular} & IMU & Yes & $\begin{array}{ll}\text { Not } & \text { re } \\
\text { quired, } & \\
\text { except } & \text { for } \\
\text { DR } & \end{array}$ & $\begin{array}{l}\text { Measures } \\
\text { RSSI, } \\
\text { performs } \\
\text { DR }\end{array}$ & Low & $5 \mathrm{~m}$ \\
\hline SAIL[16] & \begin{tabular}{|ll} 
DR & as \\
sisted & \\
trilat. &
\end{tabular} & IMU & Yes & $\begin{array}{ll}\text { Not re } \\
\text { quired, } \\
\text { except } \\
\text { DR } & \end{array}$ & $\begin{array}{l}\text { Measures } \\
\text { RSSI, } \\
\text { performs } \\
\text { DR }\end{array}$ & $0.20 \%$ & $2.3 \mathrm{~m}$ \\
\hline Kraxberger[11] & Tritat. & $\begin{array}{l}\text { Packet } \\
\text { sniffers }\end{array}$ & No & No & None & None & Unknown \\
\hline Li[12] & Distance. & None & No & No & $\begin{array}{l}\text { Can be } \\
\text { avoided }\end{array}$ & None & $6 \mathrm{~m}$ \\
\hline
\end{tabular}

has the best positioning accuracy, it requires frequency hopping, that may pose more problems with additional drivers on the user-side and increase channel inter-ference.

Overall, we see that positioning no longer requires the presence of multiple AP. This can enable new opportunities for WLAN based positioning systems and allows for improved accuracy in the case when more than one AP is present.

\section{REFERENCES}

[1] (2015). Analyzing Shopper's Behavior through WiFi Signals. Proceedings of the 2nd workshop on Workshop on Physical Analytics - WPA '15, pages 13-18.

[2] (2017). Wireless RSSI fingerprinting localization.

[3] Chapre, Y., Ignjatovic, A., Seneviratne, A., and Jha, S. (2014). CSI-MIMO: Indoor Wi-Fi fingerprinting system. Proceedings - Conference on Local Computer Networks, LCN, pages 202-209.

[4] Chilipirea, C., Petre, A. C., Dobre, C., and Van Steen, M. (2016). Presumably simple: Monitoring crowds using WiFi. In Proceedings - IEEE International Conference on Mobile Data Management, volume 2016-July, pages 220-225.

[5] Fan, Q., Zhang, D., Wu, H., and Tan, K.-L. (2016). A General and Parallel Platform for Mining Co-Movement Patterns over Large-scale Trajectories. VLDB Endowment, $10(4): 313-324$.

[6] Farid, Z., Nordin, R., and Ismail, M. (2013). Recent advances in wireless indoor localization techniques and system. Journal of Computer Networks and Communications, 2013:1-12. 
[7] Figuera, C., Mora-Jim’enez, I., Guerrero-Curieses, A., Rojo-A’lvarez, J. L., Ev-erss, E., Wilby, M., and Ramos-L'opez, J. (2009). Nonparametric model com- parison and uncertainty evaluation for signal strength indoor location. IEEE Transactions on Mobile Computing.

[8] He, S. and Chan, S.-H. G. (2016). Wi-fi fingerprint-based indoor positioning: Recent advances and comparisons. IEEE Communications Surveys \& Tutorials, 18(1):466490.

[9] Hossain, A. M. and Soh, W.-S. (2015). A survey of calibration-free indoor positioning systems. Computer Communications, 66:1-13.

[10] Kaemarungsi, K. and Krishnamurthy, P. (2012). Analysis of wlans received signal strength indication for indoor location fingerprinting. Pervasive and Mobile Computing, 8(2):292-316.

[11] Kraxberger, S., Lackner, G., and Payer, U. (2010). Wlan location determination without active client collaboration. In Proceedings of the 6th International Wireless Communications and Mobile Computing Conference, pages 1188-1192. ACM.

[12] Li, H., Sun, L., Zhu, H., Lu, X., and Cheng, X. (2014). Achieving privacy preservation in WiFi fingerprint-based localization. In Proceedings - IEEE IN- FOCOM.

[13] Liu, H., Darabi, H., Banerjee, P., and Liu, J. (2007). Survey of wireless indoor positioning techniques and systems. IEEE Transactions on Systems, Man, and Cybernetics, Part C (Applications and Reviews), 37(6):1067-1080.

[14] Lui, G., Gallagher, T., Li, B., Dempster, A. G., and Rizos, C. (2011). Dif- ferences in RSSI Readings Made by Different Wi- Fi Chipsets: A Limitation of WLAN Localization. pages 53-57.

[15] Makki, A., Siddig, A., Saad, M., and Bleakley, C. (2015). Survey of wifi posi- tioning using time-based techniques. Computer Networks, 88:218-233.

[16] Mariakakis, A. T., Sen, S., Lee, J., and Kim, K.-H. (2014). Sail: Single access pointbased indoor localization. In Proceedings of the 12th annual international conference on Mobile systems, applications, and services, pages 315-328. ACM.

[17] Mrindoko, N. R. and Minga, L. M. (2016). A Comparison Review of Indoor Positioning Techniques. International Journal of Computer (IJC), 21(1):42-49.

[18] Sapiezynski, P., Stopczynski, A., Gatej, R., and Lehmann, S. (2015). Tracking Human Mobility Using WiFi Signals. PloS one, 10(7):e0130824.

[19]Sapiezynski, P., Stopczynski, A., Wind, D. K., Leskovec, J., and Lehmann, S. (2016). Inferring Person-to-person Proximity Using WiFi Signals.

[20] Sen, S., Lee, J., Kim, K.-H., and Congdon, P. (2013). Avoiding multipath to revive inbuilding wifi localization. In Proceeding of the 11th annual international conference on Mobile systems, applications, and services, pages 249-262. ACM. 
International Journal of Wireless \& Mobile Networks (IJWMN) Vol. 10, No. 3, June 2018

[21] Vasisht, D., Kumar, S., and Katabi, D. (2016). Decimeter-level localization with a single wifi access point. In 13th USENIX Symposium on Networked Sys- tems Design and Implementation (NSDI 16), pages 165-178.

[22] Vo, Q. D. and De, P. (2016). A survey of fingerprint-based outdoor localization. IEEE Communications Surveys \& Tutorials, 18(1):491-506.

[23] Xiao, J., Zhou, Z., Yi, Y., and Ni, L. M. (2016). A Survey on Wireless Indoor Localization from the Device Perspective. ACM Computing Surveys, 49(2).

[24] Yassin, M. and Rachid, E. (2015). A survey of positioning techniques and location based services in wireless networks. In Signal Processing, Informatics, Communication and Energy Systems (SPICES), 2015 IEEE International Con- ference on, pages 1-5. IEEE.

[25] Youssef, M. A. and Agrawala, A. (2007). Analysis of the Optimal Strategy for Wlan Location Determination Systems. International Journal of Modelling \& Simulation.

[26] Z aruba, G. V., Huber, M., Kamangar, F., and Chlamtac, I. (2007). Indoor location tracking using rssi readings from a single wi-fi access point. Wireless networks, 13(2):221-235.

[27] Zheng, Y. (2015). Trajectory Data Mining: An Overview. ACM Trans. Intell. Syst. Technol. Article, 6(29). 